\title{
Dynamical Mean Field Theory of the Antiferromagnetic Metal to Antiferromagnetic Insulator Transition
}

\author{
R. Chitra and G. Kotliar \\ Serin Physics Laboratory, Rutgers University, Piscataway, NJ 08855, USA
}

(July 16, 2018)

\begin{abstract}
We study the antiferromagnetic metal to antiferromagnetic insulator using dynamical mean field theory and exact diagonalization methods. We find two qualitatively different behaviors depending on the degree of magnetic correlations. For strong correlations combined with magnetic frustration, the transition can be described in terms of a renormalized slater theory, with a continuous gap closure driven by the magnetism but strongly renormalized by correlations. For weak magnetic correlations, the transition is weakly first order.
\end{abstract}

PACS numbers: $71.30 .+\mathrm{h}$

The correlation driven metal insulator transition (MIT) or Mott transition is one of the central problems of condensed matter physics. For generic band structures, it occurs at a finite value of the interaction strength and constitutes a genuinely non perturbative phenomena. Recently, a great deal of progress has been made using the Dynamical Mean Field approach (DMFT), a method which becomes exact in the well defined limit of infinite lattice coordination [1.22]. However, all studies so far, have been confined to the paramagnetic metal $(\mathrm{PM})$ to paramagnetic insulator (PI) transition. In this letter, we study the transition from the antiferromagnetic metal (AM) to an antiferromagnetic insulator (AI) within DMFT. The motivation for this work is twofold. Experimentally, the interaction or pressure driven MIT

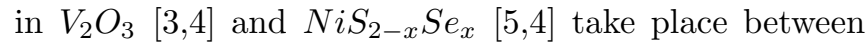
magnetically ordered states. While we are still quite far from a realistic modeling of these oxides, (in this work we only consider commensurate magnetic order and ignore orbital degeneracy and realistic band structure) we would like to take into account the effects of magnetic long range order present in these systems. On the theoretical side, we would like to understand how magnetic correlations, which controls the scale at which the spin entropy is quenched, affect the MIT.

We consider the Hamiltonian,

$$
H=t_{1} \sum_{n n} c_{i}^{\dagger} c_{j}+t_{2} \sum_{n n n} c_{i}^{\dagger} c_{j}+U \sum_{i} n_{i \uparrow} n_{i \downarrow}-J \sum_{n n} \mathbf{S}_{i} . \mathbf{S}_{j}
$$

$t_{1}$ and $t_{2}$ are nearest and next nearest neighbor hoppings respectively and $U$ is the onsite coulomb repulsion. $\mathrm{J}$ is a ferromagnetic spin spin interaction that we add to the model in order to independently control the strength of the antiferromagnetic correlations of the system $|8|$. We parametrise the hoppings as $t_{1}^{2}=(1-\alpha) t^{2}$ and $t_{2}^{2}=\alpha t^{2}$ where $\alpha$ is the degree of frustration. $t_{2}$ breaks the perfect nesting characteristic of the bipartite lattice [6,7] and allows for a direct $\mathrm{AM}$ to $\mathrm{AI}$ transition.

Within the dynamical mean field approximation, in the presence of magnetic order, the single particle Green's function on the $A$ and $B$ Neel sublattices has the following form

$G_{\sigma}^{-1}=\left(\begin{array}{cc}i \omega_{n}+\mu_{\sigma}-\tilde{\epsilon}_{k}-\Sigma_{A \sigma} & -\epsilon_{k} \\ -\epsilon_{k} & i \omega_{n}+\mu_{-\sigma}-\tilde{\epsilon}_{k}-\Sigma_{B \sigma}\end{array}\right)$

$\sigma=1,2$ refer to the spin up and down states, $m$ the staggered magnetisation and $\mu_{\sigma}=\left(\mu-\frac{U}{2}+(-1)^{\sigma} \mathrm{Jm}\right)$. $\Sigma$ are the self energies which are momentum independent within this approximation. The local Green's functions $G_{i i \sigma}$ depend on the sublattices (A and B) and obey $G_{A 1}=G_{B 2} \equiv G_{1}, G_{A 2}=G_{B 1} \equiv G_{2}$. Similarly, $\Sigma_{A 1}=\Sigma_{B 2} \equiv \Sigma_{1}$ and $\Sigma_{A 2}=\Sigma_{B 1} \equiv \Sigma_{2}$.

Within DMFT, these local self-energies and Greens functions are obtained from an Anderson impurity model

$$
\begin{aligned}
H_{i m p}= & \sum_{l \sigma} \epsilon_{l \sigma} d_{l \sigma}^{\dagger} d_{l \sigma}+\sum_{l \sigma} V_{l \sigma}\left(d_{l \sigma}^{\dagger} f_{\sigma}+\text { h.c. }\right) \\
& -\mu_{\sigma} \sum_{\sigma} f_{\sigma}^{\dagger} f_{\sigma}+U n_{f \uparrow} n_{f \downarrow}
\end{aligned}
$$

describing an impurity electron $f$ hybridising with a bath of conduction electrons $d$ via the spin-dependent hybridisation functions

$$
\Delta_{\sigma}\left(i \omega_{n}\right)=\sum_{l} \frac{V_{l \sigma}^{2}}{i \omega_{n}-\epsilon_{l \sigma}}
$$

where $\omega_{n}$ are the Matsubara frequencies. The $\Delta_{\sigma}$ obey self consistency conditions which can be expressed in terms of $G_{1}, G_{2}$ and the non-interacting density of states [2]. We restrict ourselves to the Bethe lattice in the following. The non-interacting density of states on the Bethe lattice is given by $D(\epsilon)=\frac{2}{\pi D^{2}} \sqrt{D^{2}-\epsilon^{2}}$ with $D=\sqrt{2} t$ being the half bandwidth. The self-consistency conditions now have a simple form

$$
\Delta_{\sigma}\left(i \omega_{n}\right)=\frac{t_{1}^{2}}{2} G_{-\sigma}\left(i \omega_{n}\right)+\frac{t_{2}^{2}}{2} G_{\sigma}\left(i \omega_{n}\right)
$$

We consider only the half-filled case, which on the Bethe lattice is achieved when $\mu$ is chosen to be $U / 2$ due to a 
special particle hole symmetry of the impurity problem : $d_{l \sigma}^{\dagger} \rightarrow d_{l-\sigma}, d_{l \sigma} \rightarrow d_{l-\sigma}^{\dagger}, f_{\sigma} \rightarrow f_{-\sigma}^{\dagger}, f_{l \sigma}^{\dagger} \rightarrow f_{-\sigma}$ and with $(\epsilon, V)_{l \sigma} \rightarrow-(\epsilon, V)_{l-\sigma}$ provided $\mu=U / 2$.

To study these equations we use the algorithm of Ref. [9] which uses zero temperature Lanczos method to compute the Green's functions of the impurity model given by (4) and iterate the model until the self-consistency given by (7) is achieved. Though the results to be presented were obtained for the case where the bath was represented by 5 sites, several of them were checked for $N=7$ to establish the results seen for $N=5$. The typical number of iterations varied between $50-65$ for robust convergence. We found substantially different behavior in the cases of strong and weak correlations (induced by a quenching of antiferromagnetism mimicked by ferromagnetic interactions in this paper) which we discuss separately.

\section{A. Strong Magnetic Correlations}

For small U, the system is in the paramagnetic phase. As $U$ increases beyond a critical $U_{c m}$, antiferromagnetic moments develop and the up and down spectral functions $\rho_{\sigma}(\omega)=(-1 / \pi) \operatorname{Im} G_{\sigma}(\omega)$ are no longer equal. The spin up spectral function has more spectral weight in the upper Hubbard band than in the lower Hubbard band and vice versa for the down spin spectral function. The low frequency peak in $\rho_{\sigma}(\omega)$ is no longer centered around $\omega=0$ but is split into two peaks centered around some $\pm \omega_{0}$ with a minimum at $\omega=0$. This can be attributed to the fact that the effective staggered magnetic field that is generated when antiferromagnetism develops splits the quasiparticle bands. As $U$ is increased further, the density of states at $\omega=0$ decreases until a critical $U_{M I T}$ where $\rho_{\sigma}(\omega=0)=0$ and the system becomes insulating. For $U \geq U_{M I T}$, a gap $\Delta$ which grows with $U$, opens continuously.

A plot of the staggered magnetization $m$ versus $U$ is shown in Fig. 1. $U$ is in units of $t$ in all the graphs. $m$ increases monotonically with $U$ and does not exhibit any special feature at the MIT. Note that though the magnetic moment is quite large at the MIT and increases with increasing $t_{2} / t_{1}$ it saturates only when one is well into the insulating phase. In the presence of magnetic long range order, the self energy does not show any anomalous behavior across the MIT. This should be contrasted with the paramagnetic case where $\operatorname{Im} \Sigma$ diverges as $\left(i \omega_{n}\right)^{-1}$ at the MIT. To obtain an insight into the nature of the MIT, we use the following low energy parametrization of $\Sigma_{1}$ and $\Sigma_{2}$ in conjunction with the numerical results

$$
\begin{aligned}
& \Sigma_{1}\left(i \omega_{n}\right)=h+\left(1-\frac{1}{z}\right) i \omega_{n} \\
& \Sigma_{2}\left(i \omega_{n}\right)=-h+\left(1-\frac{1}{z}\right) i \omega_{n}
\end{aligned}
$$

where $h$ is the staggered field generated by the interactions in the antiferromagnetic phase. $z$ goes to zero at the PM-PI transition, but in the presence of magnetism it is non-monotonic and remains non-zero even in the insulating phase (cf. Fig,1).

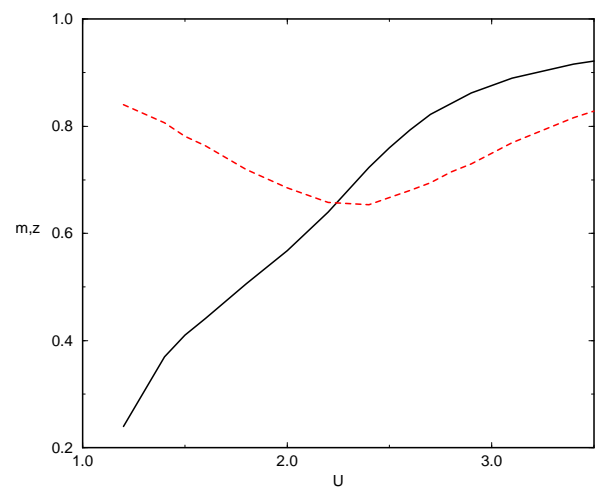

FIG. 1. The magnetization $m$ (bold line) and $z$ (dashed line) versus $\mathrm{U}$ for $t_{2} / t_{1}=0.57$ with $U_{M I T}=3.0 t$.

In terms of the local Green's functions, $\Sigma_{\sigma}$ is determined by the following expression on the Bethe lattice

$$
G_{\sigma}^{-1}+\Sigma_{\sigma}=i \omega_{n}+\mu_{\sigma}-\frac{t_{1}^{2}}{2} G_{-\sigma}-\frac{t_{2}^{2}}{2} G_{\sigma}
$$

Rewriting (7), we obtain

$$
\begin{aligned}
& 1=\left(i \omega_{n}+\operatorname{Im} \Sigma_{1}+\operatorname{Re} \Sigma_{1}\right) G_{1}-\frac{t_{1}^{2}}{2} G_{1} G_{2}-\frac{t_{2}^{2}}{2} G_{1}^{2} \\
& 1=\left(i \omega_{n}+\operatorname{Im} \Sigma_{2}+\operatorname{Re} \Sigma_{2}\right) G_{2}-\frac{t_{1}^{2}}{2} G_{1} G_{2}-\frac{t_{2}^{2}}{2} G_{2}^{2}
\end{aligned}
$$

Using (6) in (8) and continuing to real frequencies we obtain the low frequency spectral functions

$$
\left.\rho_{1,2}(\omega)=\rho_{(} 0\right)\left[1 \pm \frac{\omega}{h z}\right]
$$

where $\rho(0)$ is the density of states at the Fermi level and is given by

$$
\rho(\omega=0)=\frac{2}{\pi D^{2}} \sqrt{D^{2}-\left(\frac{h}{\alpha}\right)^{2}}
$$

Note that $\rho(0)$ does not depend on $z$. Since $h$ increases monotonically with $U, \rho(0)$ decreases with increasing $U$ in the magnetically ordered phase. This yields a critical value $h_{c}$ where $\rho(0) \rightarrow 0$ and a gap opens continuously. This is the point at which the MIT occurs. We, therefore, see that the MIT in this case is of a very different nature and there are no Kondo resonances which disappear discontinuously at the MIT. Our results indicate that in the vicinity of $U_{M I T}, h$ increases linearly with $U$ implying that $\rho(0)$ vanishes as $\left(U_{M I T}-U\right)^{\frac{1}{2}}$ as we approach the transition from the metallic side. 
We now turn to the behavior of the coefficient of the linear term of the specific heat $\gamma$. The basic physics controlling the behavior of the specific heat in the antiferromagnetic phase is the competition between the the increase of the effective mass $m^{*}$ and the decrease of the density of states $\rho(0)$. Though $m^{*} \propto\left[1-\frac{\partial \Sigma\left(i \omega_{n}\right)}{\partial i \omega_{n}}\right]=z^{-1}$, initially increases, its increase is cut off by the staggered magnetization (cf. Fig. 11). When the staggered magnetization $m$ is large the decrease in $\rho(0)$ is the dominant effect, whereas the first effect dominates when the magnetism is weak. The latter is true in the case of the paramagnetic MIT where $m^{*}$ and hence $\gamma$ are both $\propto z^{-1}$ and diverge as $z \rightarrow 0$ at the transition. This divergence is related to the fact that there is a residual entropy in the insulating phase which results from the spin degeneracy at every site. However, such a degeneracy is lost in the case where the insulator is also an antiferromagnet. Infact using Eqs. (6, 9, 10, we find

$$
\frac{\gamma}{\gamma_{0}}=\frac{1}{z} \sqrt{D^{2}-\frac{h^{2}}{\alpha^{2}}}
$$

where $\gamma_{0}=\frac{\pi k_{B}^{2}}{3 t}$ is the specific heat coefficient of the noninteracting problem. Since $z$ remains nonzero, $\gamma \rightarrow 0$ as $\left(U_{M I T}-U\right)^{\frac{1}{2}}$ at the MIT. As anticipated, we see in Fig.2 that $\gamma$ increases with $U$ for small $m$ and decreases for larger moments.

We can generalize the above to a lattice with a realistic dispersion $E_{\mathbf{k}}=\epsilon(\mathbf{k})+\tilde{\epsilon}(\mathbf{k})$ where $\epsilon$ and $\tilde{\epsilon}$ are the contributions from the $t_{1}$ and $t_{2}$ hoppings. The Fermi surface of the non-interacting system is defined by $E_{\mathbf{k}_{F}}=0$. When the interaction $U$ is turned on, the dispersions of the

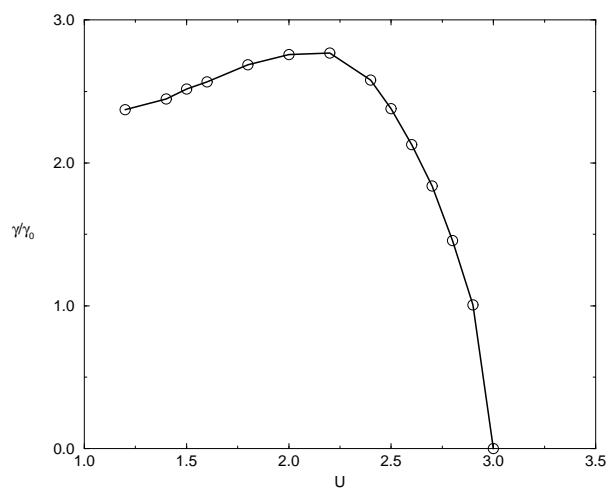

FIG. 2. $\gamma / \gamma_{0}$ vs. $U$ for $t_{2} / t_{1}=0.57\left(U_{M I T}=3.0 t\right) \cdot \gamma_{0}$ has been defined in the text.

quasiparticles change and are given by the poles of the Green's function given by Eq. 2. From the preceding analysis, we can say that the main effect of interactions is to modify the band dispersion in the following manner

$$
E_{\mathbf{k}}=z\left[\tilde{\epsilon}_{\mathbf{k}} \pm \sqrt{\epsilon_{\mathbf{k}}^{2}+h^{2}}\right]
$$

In the non-interacting case $(h=0$ and $z=1)$ the two bands overlap and there is no gap at the Fermi surface. For small $U$ in the paramagnetic phase there is no gap in spectrum but the bands are slightly renormalized by the factor $z$. When antiferromagnetic moments set in, the non-zero $h$ changes the band curvature and the renormalized bands start moving away from each other reducing the Fermi surface area. The manner in which the Fermi surface area decreases is described by the density of states $\rho(0)$. For small $h$ there are regions where the bands overlap and there is no gap in the system. At a critical value $h=h_{c}$ a gap opens up in the spectrum as the Fermi surface shrinks to zero signalling the MIT.

\section{B. Weak Magnetic Correlations: $J \neq 0$}

The previous scenario was characterized by relatively weak electron correlations, the quasiparticle residue $z$ near the transition, was at most 0.65 , indicating that a relatively high fraction of the spectral weights remain coherent. This can be understood in simple qualitative terms, when the magnetic frustration is weak, the relatively large magnetic exchange produces a large magnetization, which in turn reduces the double occupancy and hence the correlations. All the spin entropy is quenched by the spin ordering, and the presence of a hopping in the same sublattice favors coherent quasiparticle propagation. To access the strongly correlated regime where most of the spectral function is incoherent we we turn on a nonzero $J$ which reduces $h$ and $m$ and hence increases $U_{M I T}$ as suggested by (10).

We studied the case $t_{1}^{2}=0.95 t^{2}$ and $t_{2}^{2}=0.05 t^{2}$ with $t_{2} / t_{1}=0.22$. For $J=0$, this system shows the usual band MIT discussed in the previous section, in the vicinity of $U=2 t$. By choosing a $J(U)$ cf. inset of Fig. 3 , such that the magnetic moment remains very small for a large regime of $U$ as shown in Fig. 3, we move the MIT to $U=3.8 t$. In the antiferromagnetic metallic phase, a Kondo like resonance persists in the spectral function up to $U=3.8 t$ and disappears suddenly at the transition. This resonance at small $\omega$ splits into two peaks when the magnetic moment becomes appreciable as was seen earlier. However, unlike in the paramagnetic case the height of this resonance is not pinned at the value of the non-interaction density of states but decreases with increasing $U$. We also find that the slope $z$ decreases to values much smaller than was seen in the band transition picture as seen in Fig. 3. $z$ decreases until the moment becomes sufficiently large as to stop its reduction. In the insulator, which still resembles the band-insulator in that there is a pseudogap, the self-energy is still analytic and one can still define a $z$. 


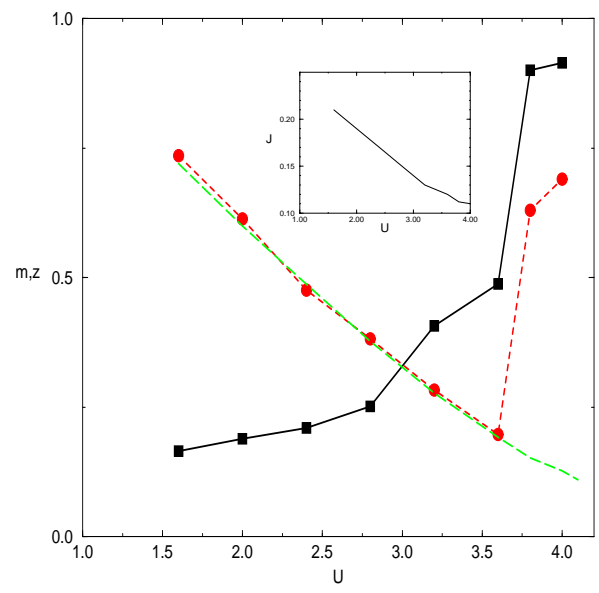

FIG. 3. $m$ (bold line) and $z$ (dashed line) vs. $U$ for the case of small frustration $t_{2} / t_{1}=0.2$ with $J$ varying as a function of $U$. $U_{M I T}=3.8 t$ in this case. The inset shows the $U$ dependence of $J$.

We find that there is a jump in $z$ and $m$ at the transition in addition to the discontinuous disappearance of the resonance mentioned earlier, reminiscent of a first order transition. On re-tracing the insulating solution as function of decreasing $U$, we find that there is co-existence. The insulator survives up to $U=3.4 t$ and there is a MIT of the pure band kind at that point and the solution goes over to one which is a metallic antiferromagnet but with a much higher value of the moment $m$ and a much larger $z$. This metallic solution disappears around $U=2.4 t$.

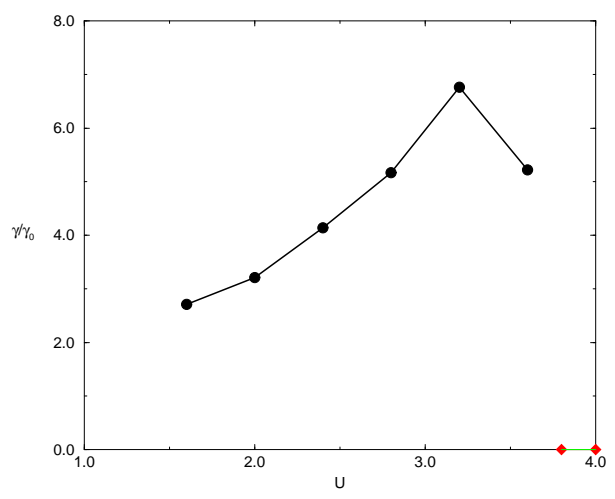

FIG. 4. $\quad \gamma$ vs. $U$ for the case of small frustration $t_{2} / t_{1}=0.2$ with $J$ varying as a function of $U\left(U_{M I T}=3.8 t\right)$.

We have measured the double occupancies in both these solutions and find that the metal with the smaller moment has higher double occupancy. This in conjunction with the ferromagnetic coupling $J$ ensures that the metal with the lower moment and higher double occupancy is the lowest energy solution. In this weak magnetic correlation regime, we find that the specific heat coefficient $\gamma$ is strongly enhanced cf. Fig. Th This scenario is reminiscent of the behavior seen in $\mathrm{V}_{2} \mathrm{O}_{3}$ [3]. Note that at the MIT, there is a finite gap which is much smaller than the Hubbard gap. Also there survive coherent quasiparticle peaks albeit with very small weight. These coherent peaks are asymmetric about $\omega=0$ in both $G_{1}$ and $G_{2}$. Close to $U=4.1 t$ these peaks vanish completely and one is left only with the incoherent Hubbard band structures. In the coexistent solution, we see that as $U$ is reduced the gap closes continuously and the the weight of these two coherent peaks increases continuously too.

To summarize, the character of the AM-AI transition is very different from the PM-PI. We found two clearly distinct scenarios depending on the strength of magnetic correlations and frustration in the system. In the limit of strong magnetic correlations, the transition takes place as a renormalized Slater transition. That is, up to a multiplicative factor which remains finite at the transition, a gap opens continuously, and $\gamma \rightarrow 0$ vanish continuously , as the MIT is approached from the metallic side. On the other hand when development of antiferromagnetic moments in the metallic phase is suppressed a new scenario emerges: the gap from the insulating side remains finite at the MIT, and a substantial increase of the specific heat is observed when the MIT is approached from the metallic side. Our results indicate that the transition is weakly first order. These two scenarios, are remarkably similar to the observations reported in $N i S_{2-x} S e_{x}$ [5,10] and $V_{2-y} O_{3}$ [3] respectively and could be developed further to interpret other experimental observations [4].

[1] W. Metzner and D. Vollhardt, Phys. Rev. Lett. 62,324 (1989).

[2] A. Georges et al, Rev. Mod. Phys. 68,13 (1996) and references therein.

[3] S. Carter et al, Phys. Rev. B. 48,16841 (1993).

[4] M. Imada, A. Fujimori and T. Tokura, Metal Insulator Transitions to appear in Rev. Mod. Phys.

[5] F. Gautier et al, Phys. Lett. 53A, 31 (1975); S. Sudo, J. Magn. Magn. Mater. 114, 57 (1992).

[6] M. Rozenberg, G. Kotliar and X.Y. Zhang, Phys. Rev. B 49,10181 (1994).

[7] D. Duffy and A. Moreo, Phys. Rev. B 55, 676 (1997).

[8] A. Georges and L. Laloux, Mod. Phys. Lett. B 11, 913 (1997).

[9] M.Caffarel and W. Krauth, Phys. Rev. Lett. 72, 1545 (1994).

[10] S. Miyasaka et al, preprint. 